\title{
Redes sociales y presencia virtual de los migrantes mexicanos en Estados Unidos
}

\section{Social networks and virtual presence of Mexican migrants in the United States}

\author{
Enrique Vaquerizo Domínguez e vd82@hotmail.com \\ https://orcid.org/0000-0002-4146-9900 \\ Universidad Camilo José Cela (España)
}

\section{Resumen}

En este artículo presento de forma parcial los resultados de mi investigación de tesis doctoral, que se ha centrado en explorar el papel de las tecnologías de la información y comunicación en los procesos de conformación identitarios y de inserción de las comunidades de migrantes en sus territorios de acogida a partir del caso de los migrantes mexicanos en Estados Unidos. Para ello realizo un mapeo de los grupos y páginas en Facebook correspondientes a la comunidad mexicana en Nueva York y Los Ángeles. Dos ciudades con trayectorias, patrones de asentamiento y estructuras organizacionales muy diferentes. En este artículo exploro la 
relación entre presencia virtual y redes sociales migratorias, y de qué forma son utilizadas las TIC en el proceso de adaptación y llegada a la sociedad de acogida por parte de las comunidades migrantes. Trato de conocer a través de un análisis comparativo tanto el grado de inclusión y participación de esas comunidades en la Sociedad de la Información y si las TIC suponen una actualización eficaz respeto a las funciones tradicionales de protección, inclusión y cohesión grupal desempeñadas por los tradicionales clubes de migrantes o por el contrario su utilización supone una respuesta a dinámicas, contextos y necesidades diferentes.

Palabras clave: TIC; clubes migrantes; comunidades virtuales; migrantes mexicanos; familias transnacionales.

\section{Abstract}

In this paper I present partially the results of my doctoral thesis research, which has focused on exploring the role of information and communication technologies in the processes of identity formation and insertion of migrant communities in their territories. Welcomed from the case of Mexican migrants in the United States. To do this I map the groups and pages on Facebook corresponding to the Mexican community in New York and Los Angeles. Two cities with very different trajectories, settlement patterns and organizational structures. In this work, I explore the relationship between virtual presence and migratory social networks, and how ICT are used in the process of adaptation and arrival in the host society by migrant communities. I try toknow through a comparative analysis both the degree of inclusion and participation of immigrant communities in the Information Society and whether ICTs representan effective update regarding the traditional functions of protection, inclusion and group cohesion with respect to traditional migrant clubs or, on the contrary, their use is a response to different dynamics, contexts and needs.

Keywords: ICT; migrant clubs; virtual communities; mexican migrants; transnational families.

Una de los rasgos característicos del fenómeno conocido como mundialización es el desarrollo exponencial experimentado durante los últimos años tanto por el sistema de transportes como de la Sociedad de la Información, acelerado por la aparición de nuevas tecnologías. Ese desarrollo ha provocado que los flujos tanto de personas como de datos sean en términos 
absolutos los mayores de la Historia. En la actualidad el mundo se mueve cada vez más y la multiplicación de interconexiones ha constreñido de forma significativa elementos antes claves del fenómeno migratorio como son el espacio y la distancia Giddens (1990), Bauman (1999), Vertovec (2009).

En este sistema marcado por una permanente movilidad e interconexión, el papel del migrante, como sujeto desarraigado y destinado a elegir entre una rápida asimilación o la marginalidad en las sociedades de acogida, ha experimentado una transformación. Hoy puede mantener los vínculos con su sociedad de origen y con las personas que continúan en ella y simultanear esos enlaces con su cotidianeidad en el nuevo destino construyendo una realidad híbrida y una identidad a la carta (Georgiu, 2010: 22), tanto en origen como de destino. La realidad transnacional, la perenne conectividad, la pertenencia cosmopolita y la flexibilidad en cuanto al mantenimiento de vínculos definirían la realidad del migrante del siglo XXI, un "migrante conectado" (Diminescu, 2008: 3) que desarrollaría una nueva modalidad de presencia, a pesar de la distancia física, posibilitada y facilitada por un patrón continuo de interacciones mediadas por las TIC (Peñaranda, 2010: 147). De esta forma las prácticas asociadas a la utilización de las TIC contribuyen hoy a su vez a la generación de nuevas formas de organización social, como son, entre otras, las familias transnacionales, haciendo posible mantener un contacto diario con familia y amigos que permanecen en el territorio de origen.

En este proceso progresivo de acortamiento de la distancia en función de la sofistificación de las nuevas TIC que aparecían en el mercado, se ha evolucionado desde el correo electrónico que permitía la multiplicación y el abaratamiento de los mensajes respecto a la modalidad postal, hasta la culminación con los servicios de mensajería instantánea como WhatsApp, Telegram o Web Msn Messenger, herramientas que permiten mantener los contactos entre las familias transnacionales de forma semigratuita y cotidiana. Del mismo modo Skype se ha convertido en una opción más barata e interactiva que el teléfono. Sin embargo, entre todas las TIC, la herramienta más completa para establecer vínculos comunitarios a gran escala y, en el caso de los nuevos migrantes, con capacidad para facilitar la llegada y establecimiento de en una sociedad de acogida serían las redes sociales, gracias principalmente a su capacidad para crear comunidades virtuales reticulares y colaborativas.

Entre esas redes sociales, debido a su propia dinámica de funcionamiento, Facebook, es la que permite un mayor nivel de instantaneidad y volumen de interacciones a sus usuarios. Estas características unidas a su facilidad de acceso, hacen de ella la red social más extendida con 2.167 millones de usuarios en todo el mundo según Hootsuite (2018), sus usuarios constituyen hoy una comunidad digital más poblada que cualquier país del planeta. 
Como señala Melella (2013), citando a Diminescu (2011), Facebook ha contribuido al desarrollo superlativo de la cultura de los vínculos, lo que extrapolado al contexto de los colectivos migrantes, les permite estar conectados con su entorno social tanto en origen como en destino. Facebook es un medio que promueve las relaciones transnacionales a través de un entorno digital caracterizadas por su volumen e inmediatez. En el ciberespacio, la consignación real a un Estado o territorio queda difuminada debido a la velocidad y multiplicidad de las interacciones que se producen en él.

\section{Objetivos}

El objetivo principal de este artículo es comparar la existencia de comunidades virtuales de migrantes, específicamente en Facebook,en las ciudades de Los Ángeles y Nueva York y relacionar esa presencia virtual con la abundancia o escasez estructuras sociales migrantes preexistentes en cada ciudad.

A partir de este objetivo principal se derivan los siguientes objetivos secundarios: reconocer ejemplos de páginas y grupos de Facebook de migrantes mexicanos en cada red social; describir tamaño, vitalidad y las características de los ejemplos más representativos, descifrar las dinámicas e interacciones que se establecen entre sus usuarios, delimitar qué servicios ofrecen y qué necesidades cubren en su respectiva comunidad migrante y explorar que otros espacios virtuales pueden canalizar la presencia de mexicanos en cada ciudad.

Los objetivos propuestos se consiguen a través de una triangulación metodológica en cuatro pasos:

1. Análisis exploratorio de las ciudades estadounidense con más índices de población mexicana en Estados Unidos

2. Análisis y medición de las federaciones de clubes migrantes que integran las estructuras sociales de ambas ciudades a partir de los datos del Instituto Nacional de Migración del Gobierno Mexicano

3. Análisis cuantitativo y cualitativo de los 10 espacios de Facebook con mayor volumen de seguidores en las dos ciudades elegidas

4. Exploración cualitativa de las publicaciones realizadas en esos espacios durante la semana del 1 al 7 de marzo de 2019. 


\section{Resultados}

Según el Anuario de Migración y Remesas mexicano facilitado por la Fundación BBVA (2018) hoy viven en Estados Unidos 49,8 millones de mexicanos, o personas de origen mexicano lo que representa el 15,2 \% de la población total estadounidense. Según este estudio los Estados que concentran un mayor índice del total de población mexicana son California (29\%), Texas (22\%), Illinois (8\%) y Nueva York (3\%).

Atendiendo a los núcleos urbanos, según datos de 2017 del Migration Policy Institute las diez principales ciudades del país con población mexicana serían las siguientes.

Tabla 1. Ciudades, población mexicana y clubes de migrantes

\begin{tabular}{|l|l|l|l|}
\hline Ciudad & $\begin{array}{l}\text { Población } \\
\text { mexicana }\end{array}$ & $\begin{array}{l}\text { Clubes de } \\
\text { migrantes }\end{array}$ & $\begin{array}{l}\text { Índice de asociación/ } \\
\text { población migrante. }\end{array}$ \\
\hline $\begin{array}{l}\text { Los Ángeles- Long Beach-Anaheim, } \\
\text { CA }\end{array}$ & 1.696 .000 & 422 & $1 / 4.018$ habitantes \\
\hline Chicago- Naperville- Elgin & 650.000 & 163 & $1 / 3.987$ habitantes \\
\hline $\begin{array}{l}\text { Houston- TheWoodlands- } \\
\text { SugarLand }\end{array}$ & 622.000 & 203 & $1 / 3.064$ \\
\hline Dallas- Fort Worth- Arlington & 613.000 & 255 & $1 / 403$ \\
\hline Riverside- San Bernardino- Ontario & 562.000 & 88 & $1 / 6.836$ \\
\hline San Diego- Carlsbad & 340.000 & 82 & $1 / 4.146$ \\
\hline Phoenix- Mesa- Scottsdale, AZ & 339.000 & 42 & $1 / 8.071$ \\
\hline New York- Newark- Jersey City & 320.000 & 28 & $1 / 11.428$ habitantes \\
\hline San Francisco, Oakland & 248.000 & 6 & $1 / 41.333$ \\
\hline McAllen-Edinburg-Mission, TX & 216.000 & - & \\
\hline
\end{tabular}

Fuente: elaboración propia a partir de los datos del Migration Policy Institute (2017)

Salvo Chicago y Nueva York, la mayoría de ciudades con una población mexicana significativa pertenecen a los estados de Texas y California, fronterizos con México y con una larga tradición de migración de una comunidad mexicana con redes sociales y estructuras consolidadas principalmente a través de clubes y asociaciones de migrantes. En ese sentido, Nueva York es junto a San Francisco la ciudad que presenta un menor tejido asociativo, 
mientras que en el otro extremo Los Ángeles, además de albergar la comunidad mexicana más numerosa, es la que cuenta también con un mayor número de clubes de migrantes.

Por tanto para este estudio he tomado como muestra las comunidades de migrantes mexicanos pertenecientes a Los Ángeles y Nueva York. Son dos de las ciudades estadounidenses que concentran un mayor volumen de migración mexicana, pero con panoramas muy diferentes en cuanto a porcentaje de población, tejido asociativo y antigüedad respecto al momento de migración.

A pesar de que la población mexicana de Nueva York es la octava del país, sin embargo es la que presenta mayores tasas de crecimiento reciente ante el estancamiento de nuevas llegadas en todo el país o incluso el crecimiento negativo Anuario de migración y remesas (2018). Sin embargo a diferencia de Los Ángeles no representan la comunidad migrante mayoritaria, según datos del CUNY (2016), los mexicanos son el tercer grupo mayoritario latino en la ciudad, con un crecimiento, entre 1990 y 2015, de 58.000 (3\% de los latinos) a 377.000 (15\%). Por el contrario, también según la ciudad de Los Ángeles alberga la comunidad mexicana más numerosa del país. Alcanza los 1,7 millones (un 15\% del total de mexicanos en el país) y sus tasas de crecimiento se han mantenido estables sobre el conjunto de la población de la ciudad. Existen más elementos diferenciadores: mientras en los Ángeles residen migrantes de segunda o tercera generación, en muchos casos bilingües o con el inglés como lengua predominante, en el caso de Nueva York se trata de una comunidad que se ha establecido en la ciudad de forma más reciente, con menores rasgos de aculturación yque, en muchos casos responde a patrones de migración circular, residiendo tan sólo unos años en territorio estadounidense antes de volver a México.

De forma consecuente, he realizado la prospección y mapeo inicial de las comunidades virtuales mexicanosen Facebook relacionadas con ambas ciudades, a través de los criterios de búsqueda relevantes como son "Mexicanos en Nueva York" y "Mexicanos en la ciudad de los Ángeles".

Tabla2. Comunidades virtuales de mexicanos en Nueva York y Los Ángeles

\begin{tabular}{|l|l|l|l|}
\hline & Páginas & Grupos & Comunidad total \\
\hline Nueva York & 5 (10.495 usuarios) & $32(68.024)$ & 78.519 \\
\hline Los Ángeles & 5 (8.323 usuarios) & 11 (7.935 usuarios) & 16.258 \\
\hline \multicolumn{3}{|c|}{ Fuente: elaboración propia a través de muestreo en Facebook. } \\
\hline
\end{tabular}


Los resultados evidencian comunidades virtuales agrupadas alrededor del factor de procedencia mucho más numerosas en el caso de Nueva York que en Los Ángeles reflejada sobre todo en el caso de los grupos frente a las páginas. Pese a la dificultad de extrapolar con exactitud estos datos a la comunidad mexicana total de la ciudad, debido a la imposibilidad de confirmar que todos sus miembros residan en Nueva York o que alguno de ellos pertenezcan a varios grupos y páginas al mismo tiempo, una estimación aproximada señalaría que el $24,5 \%$ de los mexicanos de la ciudad pertenecen a una comunidad virtual relacionada con su país de procedencia. Porcentaje que apenas alcanza el $1 \%$ en el caso de Los Ángeles.

A continuación y a partir de un periodo de observación comprendido entre el 1 y el 7 de marzo de 2019 realizaremos una clasificación de las principales comunidades virtuales de mexicanos en Nueva York y Los Ángeles, así como de sus temáticas y dinámicas de interacción fundamentales.

Tabla 3. Comunidades virtuales mexicanas estudiadas

\begin{tabular}{|c|c|c|c|c|c|}
\hline Comunidad & ID & Tipo & Miembros & Vitalidad & Temáticas \\
\hline $\begin{array}{l}\text { Mexicanos } \\
\text { en la } \\
\text { ciudad de } \\
\text { Nueva York }\end{array}$ & $\begin{array}{l}\text { groups } / 11532136514617 \\
\underline{7 I}\end{array}$ & Grupo & $\begin{array}{l}37.710 \\
\text { miembros }\end{array}$ & $\begin{array}{l}\text { Muy alta } \\
\text { participa } \\
\text { ción, } \\
32,6 \\
\text { posts } \\
\text { por día. }\end{array}$ & $\begin{array}{l}\text { Humor. } \\
\text { Información práctica } \\
\text { (búsqueda de trabajo } \\
\text { y vivienda) } \\
\text { Contenidos políticos } \\
\text { Cultura mexicana. }\end{array}$ \\
\hline $\begin{array}{l}\text { Mexicanos } \\
\text { en Nueva } \\
\text { York }\end{array}$ & $\begin{array}{l}\text { groups/25481368791140 } \\
\text { 0/ }\end{array}$ & Grupo & $\begin{array}{l}6.075 \\
\text { miembros }\end{array}$ & $\begin{array}{l}\text { Alta } \\
10,4 \\
\text { posts } \\
\text { por día }\end{array}$ & $\begin{array}{l}\text { Cultura mexicana } \\
\text { Humor } \\
\text { Política y actualidad } \\
\text { mexicana }\end{array}$ \\
\hline $\begin{array}{l}\text { Mexicanos } \\
\text { en Nueva } \\
\text { York }\end{array}$ & $\begin{array}{l}\text { https://www.facebook.co } \\
\underline{\text { m/ennewyork/ }}\end{array}$ & Página & $\begin{array}{l}711 \\
\text { seguidores }\end{array}$ & $\begin{array}{l}\text { Inactivo } \\
\text { desde } \\
2015\end{array}$ & $\begin{array}{l}\text { Información práctica, } \\
\text { locales, trabajos, } \\
\text { sitios que visitar. }\end{array}$ \\
\hline
\end{tabular}

Question, Vol. 1, N. ${ }^{\circ}$ 64, octubre-diciembre 2019. ISSN 1669-6581

Instituto de Investigaciones en Comunicación | Facultad de Periodismo y Comunicación Social | Universidad Nacional de La Plata 


\begin{tabular}{|c|c|c|c|c|c|}
\hline Mexicanos & https://www.facebook.co & Página & 3667 & Media & Entretenimientorelaci \\
\hline $\begin{array}{l}\text { en New } \\
\text { York City }\end{array}$ & $\frac{\text { m/MexicanosenNewYork }}{\underline{\text { City } /}}$ & & seguidores & $\begin{array}{l}\text { de } 4,3 \\
\text { publicac } \\
\text { iones } \\
\text { diarias. }\end{array}$ & $\begin{array}{l}\text { onado con cultura } \\
\text { mexicana. }\end{array}$ \\
\hline $\begin{array}{l}\text { Mexicanos } \\
\text { en Nueva } \\
\text { York } \\
\text { (oficial) }\end{array}$ & $\begin{array}{l}\text { https://www.facebook.co } \\
\text { m/groups/753817704696 } \\
\underline{159 /}\end{array}$ & Grupo & $\begin{array}{l}4.614 \\
\text { miembros }\end{array}$ & $\begin{array}{l}\text { Media } \\
\text { de } 29 \\
\text { publicac } \\
\text { iones } \\
\text { diarias. }\end{array}$ & $\begin{array}{l}\text { Informaciones } \\
\text { relacionadas con } \\
\text { México, intercambio } \\
\text { de información } \\
\text { práctica, humor, } \\
\text { memes } \\
\text { entretenimiento. }\end{array}$ \\
\hline $\begin{array}{l}\text { Amigos } \\
\text { mexicanos } \\
\text { de Nueva } \\
\text { York }\end{array}$ & $\begin{array}{l}\text { https://www.facebook.co } \\
\underline{\text { m/groups/261224360747 }} \\
\underline{\text { 024/ }}\end{array}$ & Grupo & $\begin{array}{l}.427 \\
\text { miembros }\end{array}$ & $\begin{array}{l}\text { Media } \\
\text { de } 6 \\
\text { postsdia } \\
\text { rios }\end{array}$ & $\begin{array}{l}\text { Contactos, } \\
\text { encuentros, } \\
\text { reuniones y } \\
\text { quedadas de la } \\
\text { comunidad mexicana } \\
\text { en la ciudad de } \\
\text { Nueva York. }\end{array}$ \\
\hline $\begin{array}{l}\text { Mexicanos } \\
\text { en Los } \\
\text { Ángeles }\end{array}$ & $\begin{array}{l}\text { https://www.facebook.co } \\
\underline{\text { m/MexicanosenLA/ }}\end{array}$ & Página & $\begin{array}{l}6.100 \\
\text { seguidores }\end{array}$ & $\begin{array}{l}\text { Media } \\
\text { de } 5 \\
\text { posts } \\
\text { diarios }\end{array}$ & $\begin{array}{l}\text { Entretenimiento, } \\
\text { programas de TV, } \\
\text { chistes y memes. }\end{array}$ \\
\hline $\begin{array}{l}\text { Mexicanos } \\
\text { Los } \\
\text { Ángeles }\end{array}$ & $\begin{array}{l}\text { https://www.facebook.co } \\
\underline{\text { m/mexicanoslosangeles/ }}\end{array}$ & Página & $\begin{array}{l}1900 \\
\text { seguidores }\end{array}$ & $\begin{array}{l}\text { Inactiva } \\
\text { desde } \\
\text { enero } \\
\text { de } 2017\end{array}$ & $\begin{array}{l}\text { Denuncias de } \\
\text { racismo y } \\
\text { discriminación, } \\
\text { publicaciones Anti- } \\
\text { Trump, nostalgia de } \\
\text { México y exaltación } \\
\text { de espíritu nacional. }\end{array}$ \\
\hline $\begin{array}{l}\text { Mexicanos } \\
\text { en Los } \\
\text { Angeles- } \\
\text { Metropolita }\end{array}$ & $\begin{array}{l}\text { https://www.facebook.co } \\
\underline{\text { m/groups/525746637461 }} \\
\underline{\text { 536/ }}\end{array}$ & Grupo & $\begin{array}{l}6300 \\
\text { miembros }\end{array}$ & $\begin{array}{l}13,4 \\
\text { posts } \\
\text { diarios }\end{array}$ & \begin{tabular}{lrr}
\multicolumn{3}{l}{ Anuncios de compra- } \\
venta productos \\
mexicanos en & Los \\
ángeles y & de
\end{tabular} \\
\hline
\end{tabular}




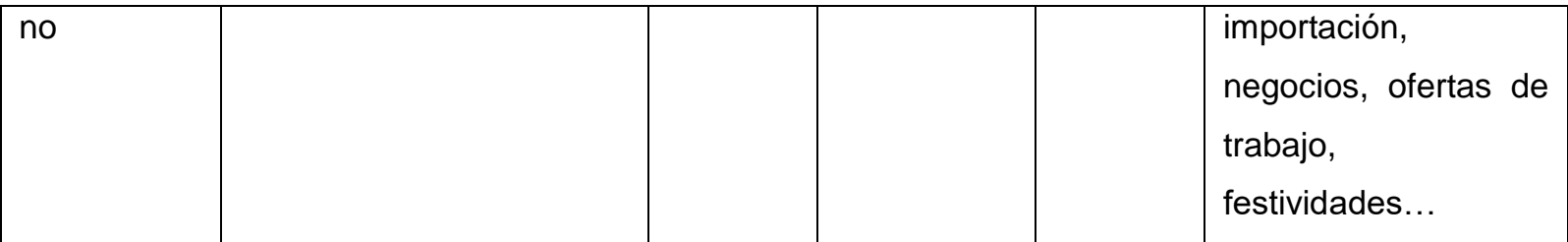

Fuente: elaboración propia a partir de muestreo en Facebook.

Al analizar estas comunidades virtuales en ambas ciudades se observa una mayor vitalidad, materializada en la frecuencia de posteo por parte de los grupos que en las páginas, la propia estructura de esta modalidad, más horizontal y participativa, en la que todos sus miembros suelen tener las mismas facultades de agregar contenido ya sea propio, curado o ajeno favorece esa capacidad de interacción. Es reseñable que la práctica totalidad de interacciones de la página se realizan en español. Del mismo modo en cuanto a temáticas los principales bloques detectados en estas comunidades serían los siguientes:

-Contenidos relacionados con el humor y en el entretenimiento: memes y vídeos sobre situaciones humorísticas, en muchos casos relacionada con el significado del hecho de ser mexicano, sus costumbres, preferencias, gustos televisivos, gastronómicos, musicales, o con determinados sucesos relacionados con la política y actualidad mexicana.

-Críticas a la política migratoria estadounidense, denuncia de situaciones de discriminación y ataques particularmente a la figura de Donald Trump.

-Información de orden práctico (ofertas de trabajo, trámites para obtener visado, alojamiento, información sobre compras de bienes básicos), en la que se involucran ciudadanos mexicanos envueltos en diferentes situaciones del proceso migratorio, aquellos que ya residen en la sociedad de acogida desde hace años o meses, los que aún no han iniciado el proceso migratorio pero buscan información antes de emprenderlo, estudiantes o simples turistas mexicanos que van a emprender una estancia corta. Este tipo de contenidos se dan con mucha más asiduidad en los grupos relacionados con Nueva York que en Los Ángeles.

-Publicidad de eventos y locales relacionados con la comunidad mexicana de cada ciudad: conciertos, restaurantes, negocios, profesionales liberales, etcétera.

-Elementos relacionados con una visión nostálgica de la sociedad que se ha dejado atrás: costumbres, música, gastronomía, festividades o la actualidad política y social.

A pesar de que la modalidad de comunidad virtual "Mexicanos en..." es la que engloba de forma más clara a los migrantes mexicanos en función de su procedencia, se han detectado otros grupos y páginas en Facebook que cumplen funciones parecidas a las anteriormente descritas, pero que engloban también al resto de la comunidad latina que vive en ambas ciudades: ya sea con una función simplemente práctica como el grupo "Trabajos en Nueva 
York" con 17.879 miembros, "Trabajos en Los Ángeles California" con 30.483 miembros o "Nueva York en español" página relacionada con la vivienda con 7.500 seguidores.

Por otra parte los propios clubes de migrantes cuentan en su práctica totalidad con presencia en Facebook, en algunos casos aglutinando algunas comunidades muy numerosas. Del mismo modo algunas páginas relacionadas con medios de comunicación latinos, en los que los contenidos mexicanos tienen especial importancia reúnen también una presencia virtual importante, casos como la página de Univisión 34 Los Ángeles con más de 800.000 seguidores - la de Telemundo 47 en Nueva York con cerca de un millón ilustran cómo los migrantes mexicanos se adscriben también a espacios virtuales de representación más amplios.

\section{Discusión}

El colectivo mexicano en Estados Unidos es una comunidad bien articulada desde el punto de vista organizacional y político. Con presencia en todos los estados del país con al menos una representación de diez mil individuos según datos del Anuario de Remesas (2018). Su activismo se enfoca en temas migratorios, ejerciendo presión con el gobierno y con el Congreso de Estados Unidos Como señala Heredia (2013: 8), en Estados Unidos existen más de 1500 clubes y organizaciones comunitarias mexicanas a través del programa de El Programa de Atención a las Comunidades Mexicanas y de los Clubes de Migrantes, fundamentalmente los vinculados con actividades eclesiales y deportivas. Un estudio realizado por González y Sánchez (2014) sobre las estructuras colaborativas y las redes sociales de los migrantes mexicanos en Estados Unidos establecía que del total de migrantes entrevistados el $40 \%$ pertenecía a algún club migrante o asociación.

A través de estas instituciones se realizaban actividades relacionadas tanto con la sociedad de acogida como la de origen: colectas para la construcción-remodelación de lugares público, reivindicaciones para lograr el derecho a voto, protección frente a las redadas de migración o apoyo económico para aquellos que han sido deportados. Del mismo modo la importancia de la red de contactos personales para hitos claves relacionados con el proceso migratorio. Según González y Sánchez (2014: 10), los migrantes suelen llegar al lugar de destino con el contacto de algún familiar y pernoctan con ellos en el $95 \%$ de los casos, el $55 \%$ habían permanecido en pisos compartidos desde su llegada, mientras que $30 \%$ se independizó al traer a su familia.

Siguiendo con González y Sánchez (2014: 7), la práctica totalidad de los migrantes cuenta con más de un amigo en el lugar donde radican en los Estados Unidos; el 70\% pertenece a su región de origen en México; mientras que el 30\% procede de otras regiones mexicanas o son latinos. Se observa cómo esas redes sociales, además de ser importantes en las primeras 
etapas del proceso migratorio para conseguir alojamiento y empleo, suelen constituir la base para su integración en actividades colectivas y sociales posteriores. Además de la vivienda en aspectos como conseguir empleo en Estados Unidos el tejido social del migrante es fundamental. Según un estudio realizado por Levine (2006: 9), el 78\% de los mexicanos residentes en Estados Unidos encuestados manifestó haber conseguido su primer empleo por medio de un pariente o un amigo.

Primero la aparición de los teléfonos móviles y más adelante la generalización de Internet y la llegada de las TIC han ayudado a fortalecer los nexos de comunicación. Hoy sirven para informarse y defenderse de las redadas, buscar empleo o vivienda así como informarse de los trámites jurídicos o administrativos necesarios en el país de acogida. Del mismo modo algunas TIC, como las comunidades virtuales estudiadas en este artículo,ayudan también a favorecer la cohesión grupal y reforzar el sentimiento de pertenencia identitaria y los vínculos con la sociedad de origen. En el caso mexicano además la aparición de Trump ha supuesto un catalizador para la expresión del movimiento asociativo mexicano en Estados Unidos, unido a través de un mayor activismo político frente a una amenaza exterior. Fenómeno que se percibe con especial claridad en redes como Facebok.

Al proceso de apropiación de las nuevas tecnologías, por parte de las comunidades migrantes, ha contribuido durante los últimos años una disminución considerable de la brecha digital a medida que lo hacían el costo de acceso a internet y se multiplicaban los soportes tecnológicos: teléfonos móviles, tabletas, laptops, etcétera. Las familias transnacionales de migrantes han visto multiplicadas sus posibilidades de interacción, no solo comunicativa sino de envíos físicos: han pasado de las empresas de envíos de paquetes para enviarse documentos, fotografías y recuerdos a hacerlo a través de las TIC, aprovechando la diversidad de contenidos y formatos que ofrecen estas: texto, fotografías, vídeos, audios...

A través de las TIC y sus posibilidades de acceso y bajo coste, se recrea una nueva experiencia de comunicación donde la calidad e intensidad del flujo se ve incrementada exponencialmente. Ya no se requieren forzosamente el ordenador o el cibercafé cuando hay smartphones económicos con megas en telefonía de prepago (Marchand y Rodríguez, 2013). Las llamadas telefónicas, los correos electrónicos, las conexiones vía Skype, los comentarios en páginas y grupos Facebook, o los chats a través de esta red social o vía WhatsApp, permiten una nueva construcción de la vida social y familiar en la distancia.

Del mismo modo autores como Komito (2011) sostienen que TIC como las redes sociales permiten generar crear capital social y vincular a personas que solo tenían conexiones breves lo que llamaríamos vínculos débiles, en el caso de los migrantes otros compatriotas a los que aún no conocen y al mismo tiempo mantener en el tiempo lazos fuertes, en este caso vínculos 
transnacionales, con amigos y familiares que han permanecido en la sociedad de origen. Resulta revelador tras los resultados de este estudio cómo la existencia de comunidades virtuales destinadas a la creación de esos vínculos débiles, es mucho más potente en una ciudad como Nueva York, donde la presencia de la población mexicana es más reciente, en rápido crecimiento y sin una estructura asociacional consolidada que en una ciudad como Los Ángeles, dónde si existe ese tejido y la migración mexicana cuenta con años de tradición.

Por otra parte las dinámicas observadas en las comunidades analizadas en este capítulo evidencian esa necesidad de desarrollar, mantener y recrear redes personales, sociales, lingüísticas o culturales a nivel transnacional Oiarzabal (2012), facilitando, principalmente en aquellos casos donde las redes colaborativas offline son más débiles, la vertebración de cierta cohesión grupal no sólo en el territorio de origen sino en el de acogida. Como señala Peñaranda (2010: 4), se ponen en juego, y se comparten diferentes elementos, significados, experiencias y recuerdos que buscan saber del otro, pero también hacerse presente en la ausencia. Con ellas los migrantes dedican una gran cantidad de esfuerzos a construir una copresencia virtual con sus familiares y amigos, mientras que ensayan formas de mantener ese contacto y, de este modo, ejercitar un cuidado familiar y afectivo transnacional.

\section{Conclusiones}

Las comunidades virtuales de migrantes suponen en muchos casos una respuesta o sustitutivo frente a la escasez de redes sociales analógicas consolidadas. Según esta investigación la presencia virtual es mucho más amplia en una ciudad como Nueva York donde escasean las asociaciones y Clubes de Migrantes, tradicional puerta de integración de los migrantes mexicanos recién llegados a Estado Unidos, que en comunidades mexicanas más consolidadas de otras ciudades como Los Ángeles. Del mismo modo, junto a esa ausencia de estructuras en el desarrollo de la presencia virtual influyen otras características como el carácter reciente y aún minoritario de esa migración mexicana dentro de la estructura migrante de cada ciudad.

En aquellas comunidades migratorias más consolidadas en el tiempo, con estructuras fuertes y migrantes ya de segunda, tercera o cuarta generación con un mayor grado de integración en la sociedad de acogida, la existencia de comunidades virtuales relacionadas con su etnicidad quedaría diluida en espacios menos específicos y relacionados con su cotidianeidad: negocios, asociaciones específicas, medios de comunicación, comunidades adscritas a un espectro étnico más amplio como el latino, entre otros. 
Las dinámicas creadas dentro de las comunidades virtuales estudiadas (grupos y páginas de Facebook) señalan que los usuarios intentan satisfacer algunas necesidades que tradicionalmente han desarrollado y desarrollan las redes interpersonales de migrantes: búsqueda de trabajo, de vivienda, información sobre trámites migratorios, defensa frente a amenazas exteriores, etcétera. Del mismo modo la propia naturaleza de estas herramientas permite un mantenimiento más efectivo de los vínculos transnacionales con la sociedad de origen ya sea a través de la nostalgia o del seguimiento de su actualidad.

A través de esta investigación no se han podido extraer resultados concluyentes sobre la efectividad de los espacios virtuales a la hora de conformar una comunidad migrante cohesionada en comparación con asociaciones tradicionales, pese a que se han observado convocatorias para encuentros, quedadas y actividades offline de la comunidad mexicana en las ciudades estudiadas, estas publicaciones no parecen ser la tónica habitual y no se conoce su efectividad. Si se intuye su potencial para un primer acercamiento de los individuos a una comunidad migrante desestructurada.

Las comunidades virtuales si resultan muy eficaces como espacio de refugio y mecanismos de defensa en entornos de presión migratoria, así como de mantenimiento de valores culturales asociados a la sociedad deorigen. Estos espacios funcionan como canales de información cuando existe un temor compartido a dejarse ver en asociaciones públicas por miedo a represalias como las deportaciones.

La mayor parte de dinámicas participativas se han observado en aquellos espacios virtuales que por su propia concepción estructural presentaban un mayor grado de horizontalidad e interactividad. Grupos frente a páginas de Facebook, en los que todos sus miembros tenían la capacidad de agregar contenidos de forma igualitaria y establecer interacciones directas.

\section{Bibliografía}

Bauman, Z. (1999). Modernidad Líquida. Ciudad de México: Fondo de Cultura Económica.

Bergard, W. L. (2016). The latino population of New York City 1990-2015. New York:_The Center for Latin American, Caribbean and Latino Studies \& City University of New York (CUNY). Recuperado de https://bit.ly/2E2HWjp

Diminescu, D. (2008). The connected migrant: an epistemological manifestó. Social Sciencelnformation, 47, 565-579. Recuperado de https://bit.ly/2E5ERis

Diminescu, D. (2011). e-Diasporas Atlas: Exploration and Cartography of Diasporas on digital network. París: Editions de la FMSH. 
Fundación BBVA Bancomer (2018). Anuario de Migración y Remesas. México. Recuperado de https://bit.ly/2t4iTds

Georgiu, M. (2010). Identity, Space and the Media: Thinking through Diaspora. Revue européenne des migrations internationales, 26(1), 17-36. Recuperado de https://journals.openedition.org/remi/5028

Giddens, A. (1990). Consecuencias de la modernidad. Barcelona: Alianza Editorial.

González, A. y Sánchez, Y. (2014). Las Redes Sociales de Migrantes Mexicanos en Estados Unidos: Una Estrategia para la sobrevivencia. Revista Cimexus,11(1), 1-17. Recuperado de https://www.cimexus.umich.mx/index.php/cim1/article/view/175

Heredia, C. (2013). Los mexicanos en Estados Unidos como actores políticos transnacionales. Revista mexicana de política exterior, 98, 167-195. Recuperado de https://bit.ly/2LFQElt

Komito, L. (2011). Social media and migration: virtual community 2.0. Journal of the American Society for Information Science and Technology, 62(6), 1075-1086. Recuperado de https://onlinelibrary.wiley.com/doi/full/10.1002/asi.21517

Levine, E. (2006). Hijos de migrantes mexicanos en las escuelas de Estados Unidos Sociológica, 21(60), 173-205. Recuperado de https://bit.ly/2PztAMr

Hootsuite (2018). Digital in 2018 Global Overviw. Recuperado de https://bit.ly/2qD52tE

Marchand, M. H. y Meza Rodríguez, E. (2013). Diásporas digitales: migrantes mexicanos y las nuevas tecnologías de comunicación. Saberes y Ciencias, 2(14), 1-8. Recuperado de https://bit.ly/38iwi1w

Melella, C. (2013). Migración y Tecnologías de la Información y de la Comunicación (TIC). La presencia de los periódicos de migrantes en Internet y los desafíos del análisis de las redes sociales virtuales. Cuadernos de $H$ ideas, 7(7), 1-18. Recuperado de http://perio.unlp.edu.ar/ojs/index.php/cps/article/view/1781

Migration Policy (2017). Mexican inmigrants in United States. Recuperado de https://bit.ly/344SLf5

Oiarzabal, P. J. (2012). Diaspora Basques and Online Social Networks: An Analysis of Users of Basque Institutional Diaspora Groups on Facebook. Journal of Ethnic and MigrationStudies, 38(9), 1469-1485. doi: 10.1080/1369183X.2012.698216

Peñaranda, M. C. (2010). "Te escuchas aquí al lado". Usos de tecnologías de la información y la comunicación en contextos migratorios transnacionales. (Tesis doctoral). Universidad Autónoma de Barcelona, Barcelona. doi: https://doi.org/10.5565/rev/athenead/v0n19.787

Peñaranda, M. C. (2011). Migrando en tiempos de globalización: usos de tecnologías de la información y la comunicación en contextos migratorios transnacionales. En García

Question, Vol. 1, N. ${ }^{\circ} 64$, octubre-diciembre 2019. ISSN 1669-6581

Instituto de Investigaciones en Comunicación | Facultad de Periodismo y Comunicación Social | Universidad Nacional de La Plata La Plata | Buenos Aires | Argentina 
Castaño, F. y Kressova, N. I Congreso Internacional sobre Migraciones en Andalucía. Resúmenes de ponencias y comunicaciones (pp. 2023-2032). Granada: Instituto de Migraciones. Recuperado de https://bit.ly/2PapNXg

Vertovec, S. (2009). Transnationalism. London \& New York: Routledge. 